\title{
The observation of abnormal signals in laser flash photolysis: A type of probable synchronized nuclear spin signals
}

\author{
Wen $\mathrm{Li}^{\mathrm{a}}{ }^{\mathrm{a}}$, Xin Hou Liu ${ }^{\mathrm{a}}$, Chen Ho Tung ${ }^{\mathrm{a}}$, Zheng Ping Hao ${ }^{\mathrm{b}}$

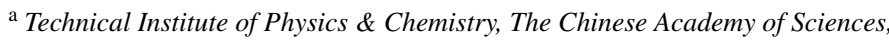 \\ Datun Road,Chaoyang District, Beijing 100101, PR China \\ ${ }^{\mathrm{b}}$ Research Center for Eco-Environmental Science, The Chinese Academy of Sciences, \\ Shuangqing Road, 18, Beijing 100085, PR China
}

Received 27 May 2005; received in revised form 22 October 2005; accepted 7 November 2005

\begin{abstract}
In laser flash photolysis (LFP) work, noise-like signals were observed together with transient absorption decay spectra in time domain. Analyzed results show that it is a valuable resonance spectrum of excited state molecules, in which four molecular cases are given here. We proposed that this kind signal might originate from nuclear or nuclear and electron spin resonance features in the excited molecules based on radio wave frequency spectrum levels and the significant interaction with static magnetic field.
\end{abstract}

(c) 2005 Elsevier B.V. All rights reserved.

Keywords: Laser flash photolysis; Transient absorption spectra; Hydrogen nuclear spin

\section{Introduction}

Recent years, in our experimental work we have frequently found that some very strong or very significant noise-like signals presented in transient absorption decays. In practice, this unexpected phenomenon most appears in two or a few wavelength decays and these signals always ride on the decay curve of $\mathrm{T}-\mathrm{T}$ absorption [1] signals. To show this point, four experimental examples chosen (adenine, cytosine, uracil and a platinum complex) were given in this work. And their molecular structures are illustrated in the molecular scheme. Here, the represent cases from the $\mathrm{T}-\mathrm{T}$ absorption decay for adenine sensitized with $\mathrm{Ru}(\mathrm{bpy})_{3} \mathrm{Cl}_{2}$ (about which the experimental results will be published late) and $\mathrm{Pt}_{2}\left[\left(\mathrm{C}_{34} \mathrm{H}_{22} \mathrm{~N}_{4}\right)\left(\mathrm{PPh}_{3}\right)_{2} \mathrm{Cl}_{2}\right] \mathrm{Cl}_{2}$ [2] were given in Fig. 1. We can see clearly that there are many spikes on the definite time range of decay curves. And the intensity of these spikes was high in some cases, but it was not always. Meanwhile, those abnormal signals often have different distribution regions both at decay time and at wavelengths for different molecules. For instance, the noise-like signal phenomena occurred at the decays of 620 and $610 \mathrm{~nm}$ (the spikes centered on the top of

\footnotetext{
* Corresponding author. Tel.: +861064867286; fax: +861064879375 .

E-mail address: zklee2@yahoo.com (W. Li).
}

curve at this wavelength) for adenine, and at the decays of 640 and $630 \mathrm{~nm}$ for the Pt complex, as shown in Fig. 1 where only 640 and $650 \mathrm{~nm}$ decays were illustrated for comparison. But the decay wavelength with the abnormal signals may have a red or blue shift due to sample temperature and other experimental conditions. However, it seems to show that the spikes could be detected during the whole time range of decays.<smiles>Nc1ncnc2[nH]cnc12</smiles>

(I)<smiles>Nc1cc[nH]c(=O)n1</smiles>

(II)<smiles>O=c1cc[nH]c(=O)[nH]1</smiles>

(III)

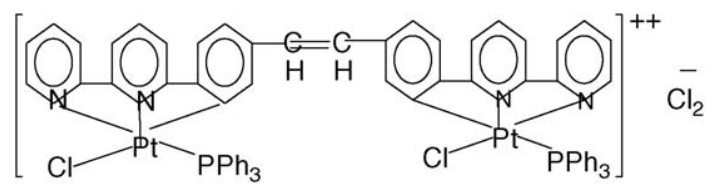

(IV)

The scheme for molecular structures 


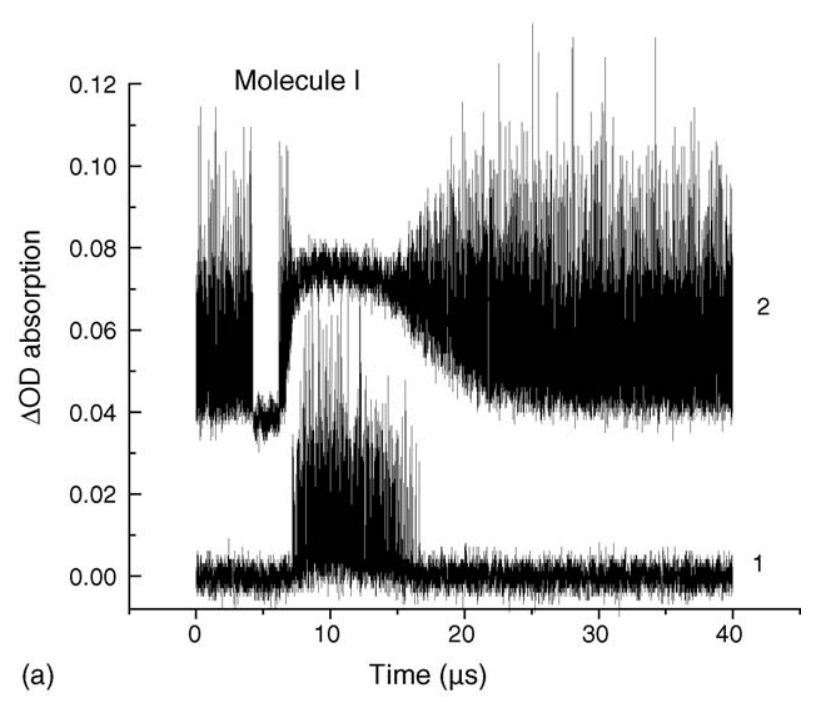

(a)

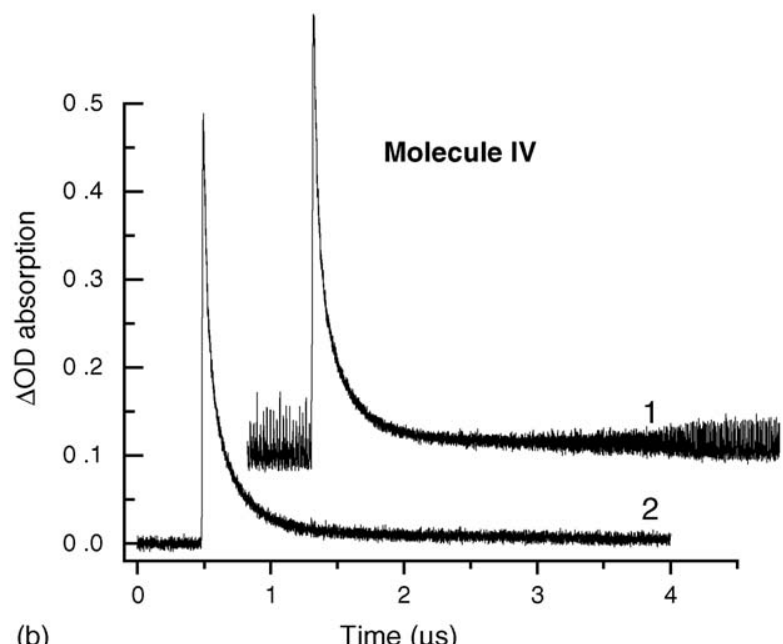

(b)

Time $(\mu s)$

Fig. 1. The transient absorption decay curves of (above) adenine sensitized with $\mathrm{Ru}(\mathrm{bpy})_{3} \mathrm{Cl}_{2}$ at $610 \mathrm{~nm}$ (1) and $620 \mathrm{~nm}$ (2) in ethanol solvent and of (below) $\mathrm{Pt}_{2}\left[\left(\mathrm{C}_{34} \mathrm{H}_{22} \mathrm{~N}_{4}\right)\left(\mathrm{PPh}_{3}\right)_{2} \mathrm{Cl}_{2}\right] \mathrm{Cl}_{2}$ at $640 \mathrm{~nm}$ (1) with noise-like signals and $650 \mathrm{~nm}$ (2) without noise-like signal in $\mathrm{C}_{2} \mathrm{H}_{4} \mathrm{Cl}_{2}$ solvent using $355 \mathrm{~nm}$ laser with about $80 \mathrm{~mJ}$.

\section{Experimental}

In the experimental work, adenine, cytosine and uracil were used as received, which were purchased from Acros Organics (NJ, USA) and Fluka BioChemika, respectively. Their purity is more than $99 \%$ (HPLC) and $>99 \%$, respectively. AR grade alcohol was purified by adding magnesium turnings reacting with water, refluxing under boiling for $3 \mathrm{~h}$ and distilling before use. The concentration of all samples was about $2 \times 10^{-5} \mathrm{M}$ in alcohol. Before measurement each sample was deoxygenated for at least $30 \mathrm{~min}$ by bubbling through the solution with high purity nitrogen gas $(99.999 \%)$. The sensitizer $\mathrm{Ru}(\mathrm{bpy})_{3} \mathrm{Cl}_{2}$ used for molecules I-III was a gift from a friend Dr. J.Y. Xu who is at Shanghai Institute of Organic Chemistry, The Chinese Academy of Sciences. The Pt compound and $\mathrm{C}_{2} \mathrm{H}_{4} \mathrm{Cl}_{2}$ solvent were spectra grade.

In order to make the phenomenon more understandable, the scheme-measured is shown in Fig. 2, including permanent mag-

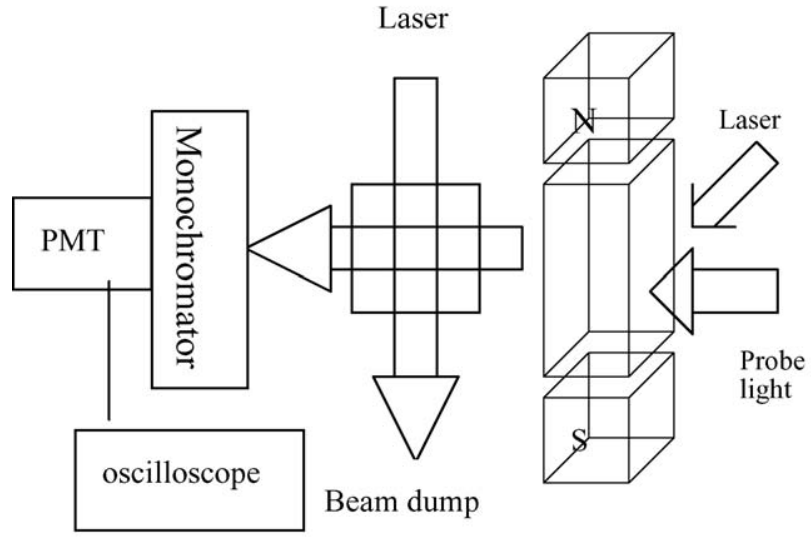

Fig. 2. The top view from sample cell in normal case and side view from sample cell with a permanent magnetic field in LFP.

netic field case. And also the measurement system is described in detail here. It is a conventional LFP instrument set-up named as LP920 by Edinburgh Instruments Ltd. (England, UK). A ns Nd:YAG laser system was used for sample excitation (Surelite Continuum laser, pulse width 5-8 ns) and Xe lamp with $450 \mathrm{~W}$ was used as probe light. The conversion of light to electricity was completed with the HAMAMATSU PMT R955. Laser light was inducted into a sample quartz cell $(1 \mathrm{~cm} \times 1 \mathrm{~cm} \times 4 \mathrm{~cm})$ by right angle direction with probe light on the plane of the optical stable, and finally the signals were recorded with a Tektronix digital $100 \mathrm{MHz}$ TDS3012B oscilloscope. The other point we should mention is the timing sequence in the LFP system different from other conventional LFP system. In the LP920 system all timing and delays were controlled relative to the probe light pulse, that is, exciting laser must superpose to an available platform of the Xe lamp pulse with about $4 \mathrm{~ms}$ time limit. The transient absorption signals were measured directly on the Xe lamp pulse platform as backgrounds and the correction of baselines was made automatically in the program. Here, a point we should keep in mind is that transient absorption signal rising may not be a start point of forming excited state due to different delays for the oscilloscope and Q-switch triggers. So, that is the reason why the noise-like signal can occur often before transient absorption signal appearing.

\section{Results and discussion}

For the mentioned abnormal experimental cases above we are sure that those spikes should be a kind of signals from the studied sample. Moreover, the noise-like signal does not be an accidental phenomenon but it is always encountered in our LFP work so long as suitable conditions are maintained, such as sampling rates and laser intensity, sample temperature, etc. Hence, we attempted to analyze the unusual phenomenon, and we considered converting these data into frequency domain by fast Fourier transform (FFT) method [3] due to intuitional undiscerning of signals in time domain. The frequency range by FFT method is determined readily in terms of sampling rate [4] in an experimental work, such as $2.5 \times 10^{8} \mathrm{~Hz}$. Due to the conjugative properties 


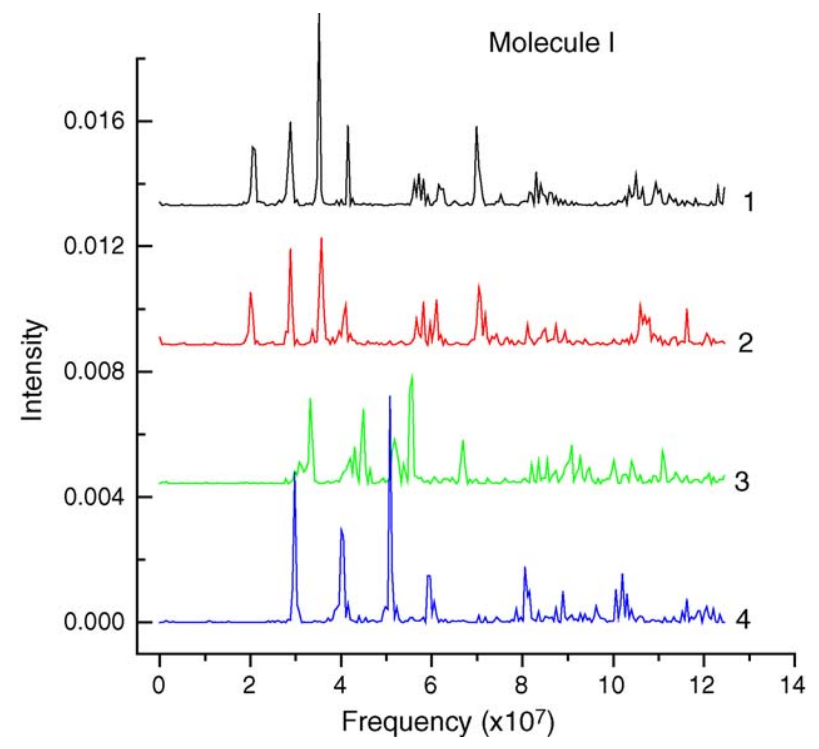

Fig. 3. The FFT results are (1) from 0 to 2.048, (2) from 2.052 to 4.092 , (3) from 26.000 to 28.044 and (4) from 36.000 to $38.044 \mu \mathrm{s}$ of the time domain data at $620 \mathrm{~nm}$ for the adenine sample.

of FFT results the frequency range was chosen only from 0 to its corresponding half.

All calculations were carried out using a program self-edited. Firstly, a series of FFT results were obtained through using sequential each interval with 512 data points taken from the abnormal data of the adenine sample and sampling rate is $2.5 \times 10^{8} \mathrm{~Hz}$ in this case. The typical spectra are shown in Fig. 3 . Note that the usual strong peak at origin point was cancelled for all spectra due to no physical meaning. Most results reveal that there are similar or almost same spectra with five stronger peaks and some small peaks, especially for initial and final time domain period of the decay. From their frequency positions the energy levels of the peaks are in the range of radio waves in agreement with the frequency range of NMR in spite of different from its conventional NMR spectra [5]. Here, we only may infer that they might be transient ${ }^{1} \mathrm{H}$ NMR spectra of the excited state of adenine and the three peaks with a similar triplet may be assigned to $\mathrm{H}$ atoms bonding to $\mathrm{C}$ atoms due to two neighbor $\mathrm{N}$ atoms with some nuclear spins and the others assigned to $\mathrm{H}$ atoms bonding to $\mathrm{N}$ atoms. However, whether these spectra are ${ }^{1} \mathrm{H}$ NMR of the first triple state $\left(\mathrm{T}_{1}\right)$ of adenine further evidences will be needed afterwards. Moreover, if the peaks will be assigned accurately the external or internal standard reference also need be established. In addition, some spectra with which the time domains are towards central time intervals have significant changes for both their peak positions and strengths progressively. In view of theoretical dynamics [6], spectroscopic transition process is a continuous changing process of linear superposition coefficient ratio for initial and final state wave functions. Hence, most possible same states are those from initial and final periods of the decay and the results from molecule (I) exactly show this point. But, $\mathrm{T}_{2}$ triplet state that is near to the top of $\mathrm{T}-\mathrm{T}$ absorption decays did not have relatively stable spectra of the kind. So, the features of $T_{1}$ wave function gradually diminishing and the strength decreasing towards the top of the decay just show the
U-shaped change during the absorption process in accord with intensity changing of our frequency spectra obtained.

To test and verify above results using further samples, same analyses will be done for similar abnormal cases of the other two molecules (cytosine and uracil) based on the above analysis for molecule I. Fig. 4 illustrates the FFT spectra for the cytosine. Clearly, the two spectra at initial time stage are nearly same. But, the other spectrum with only one stronger peak seems to change greatly. The possible reasons may be due to the tautomer of cytosine that the $\mathrm{H}$ on the neighbor $\mathrm{N}$ transforms to the $\mathrm{O}$ atom forming a hydroxyl group with stronger ${ }^{1} \mathrm{H}$ NMR signal. In similar situation, we also observed the case of uracil sample, shown as molecular structure (III). The related frequency spectra were shown in Fig. 5. In this example, basically there are significant changes in both before signal rising and its decay tail region. First, we can see that the two spectra are not same from $0-2.048$ to $2.052-4.092 \mu \mathrm{s}$, and the triplet peak in Fig. 5 (above) (1) changes into a double peak in (2). Moreover, after signal rising region the spectra have a big change than that before signal rising region but basically their spectra centered at about $8 \times 10^{7} \mathrm{~Hz}$ are similar within this time range. In addition, the abnormal signal was appeared in same time domain at different proximate wavelength regions in which there were different
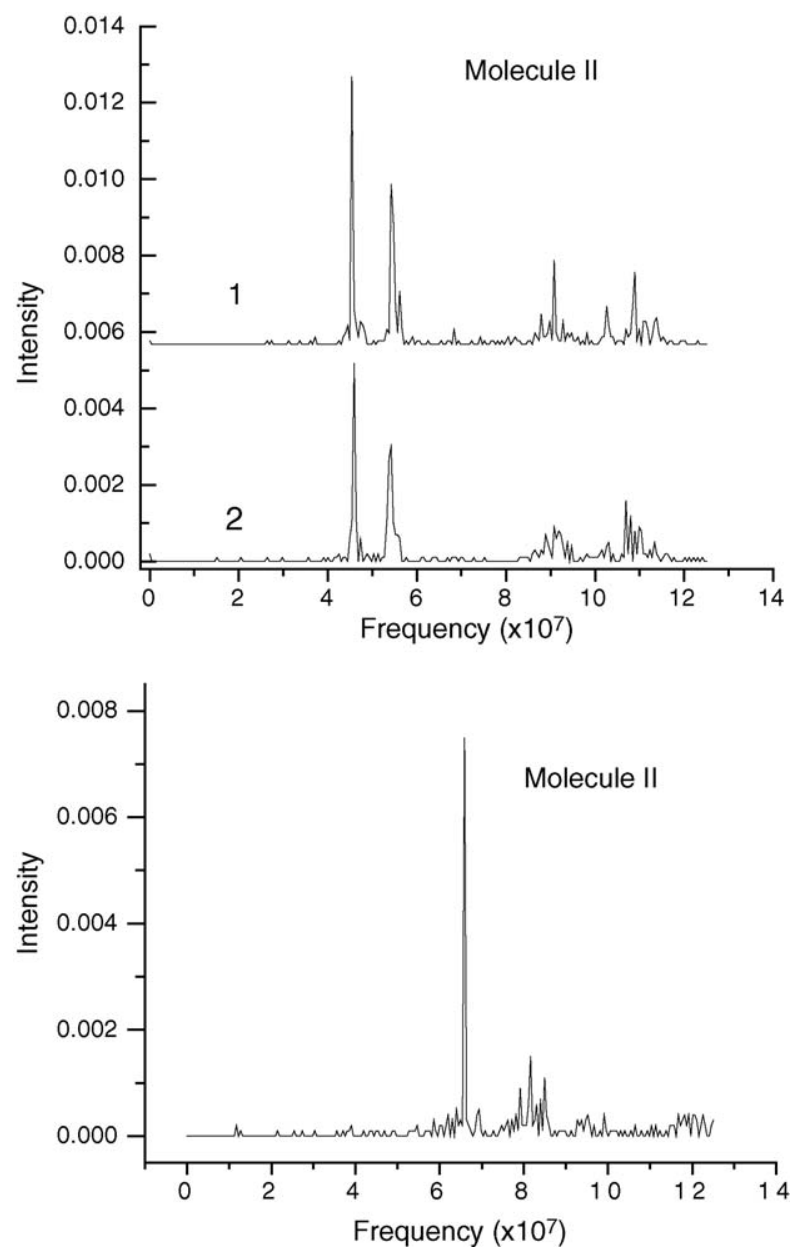

Fig. 4. The spectra are above (1) from 0 to $2.048 \mu$ s and (2) from 2.048 to $4.096 \mu$ s and below from 25.140 to $27.184 \mu$ s at $650 \mathrm{~nm}$. 

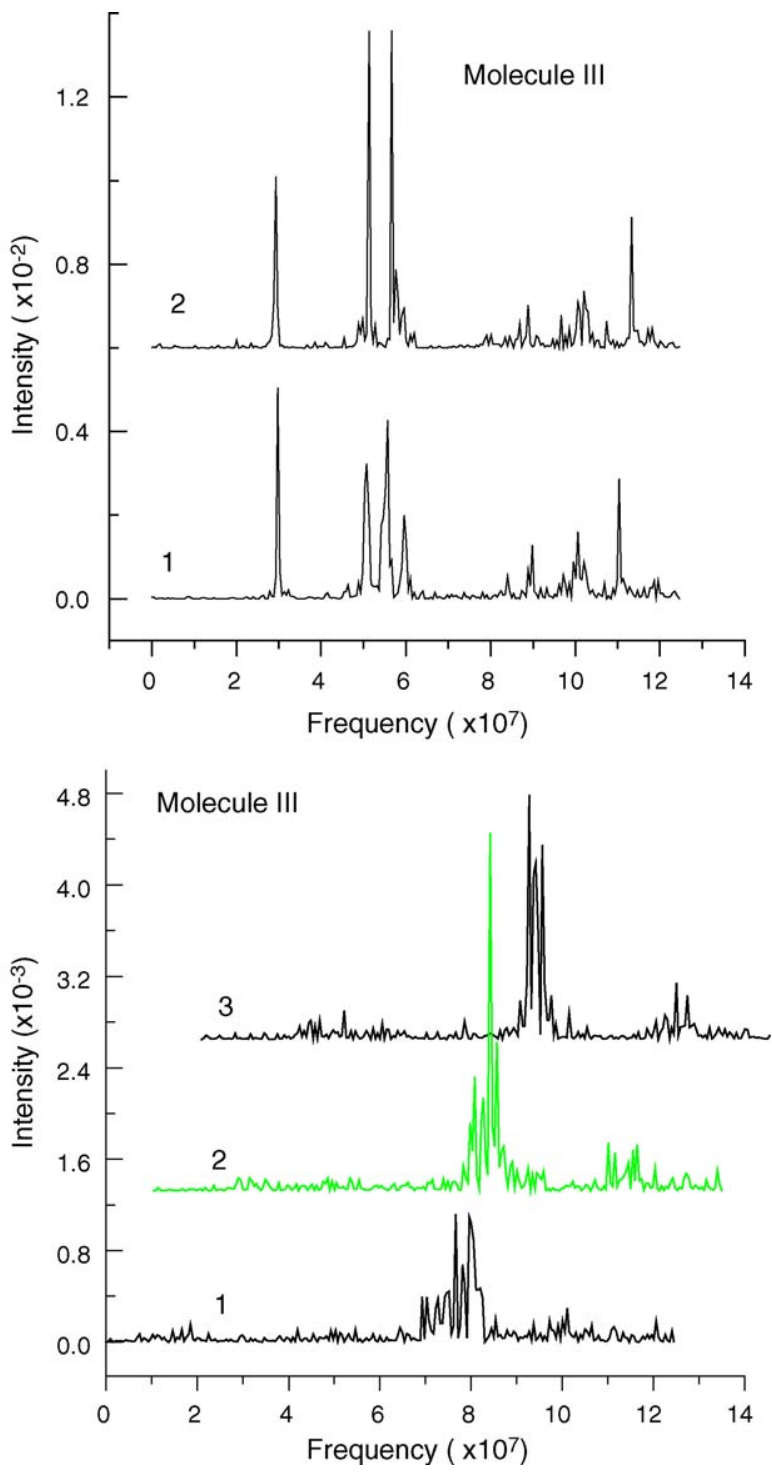

Fig. 5. The FFT results are from $540 \mathrm{~nm}$ above (1) from 0 to 2.048 and (2) from 2.052 to $4.092 \mu \mathrm{s}$ and below (1) $30.192-32.236$, (2) $34.288-36.332$ and (3) $36.328-38.372 \mu \mathrm{s}$.

spectra, shown as in Fig. 6 (above). However, there was only this example with the phenomenon in the four examples. Seen from the Fig. 6 (above), at $540 \mathrm{~nm}$ decay curve, the abnormal signal analysis shows that there are more frequency peaks but those peaks decrease with increasing wavelength all at the beginning time region with 512 channels. This may be caused by the different tautomer from uracil due to two oxygen atoms in molecule (III). However, there are almost similar spectra after signal rising region, shown as Fig. 6 (below). So, we may assume that they were from a tautomer within that time domain. In other words, the tautomer should be relative stable configuration during the period of the exciting state decay process.

Finally, let us see the last example as shown in Fig. 7 for the Pt complex. The intensity of spike signals is much less than its T-T absorption signal and the FFT spectrum patterns almost are not changeable. Here, at $640 \mathrm{~nm}$ for the Pt complex, we could expect that the transient absorption perhaps focused on
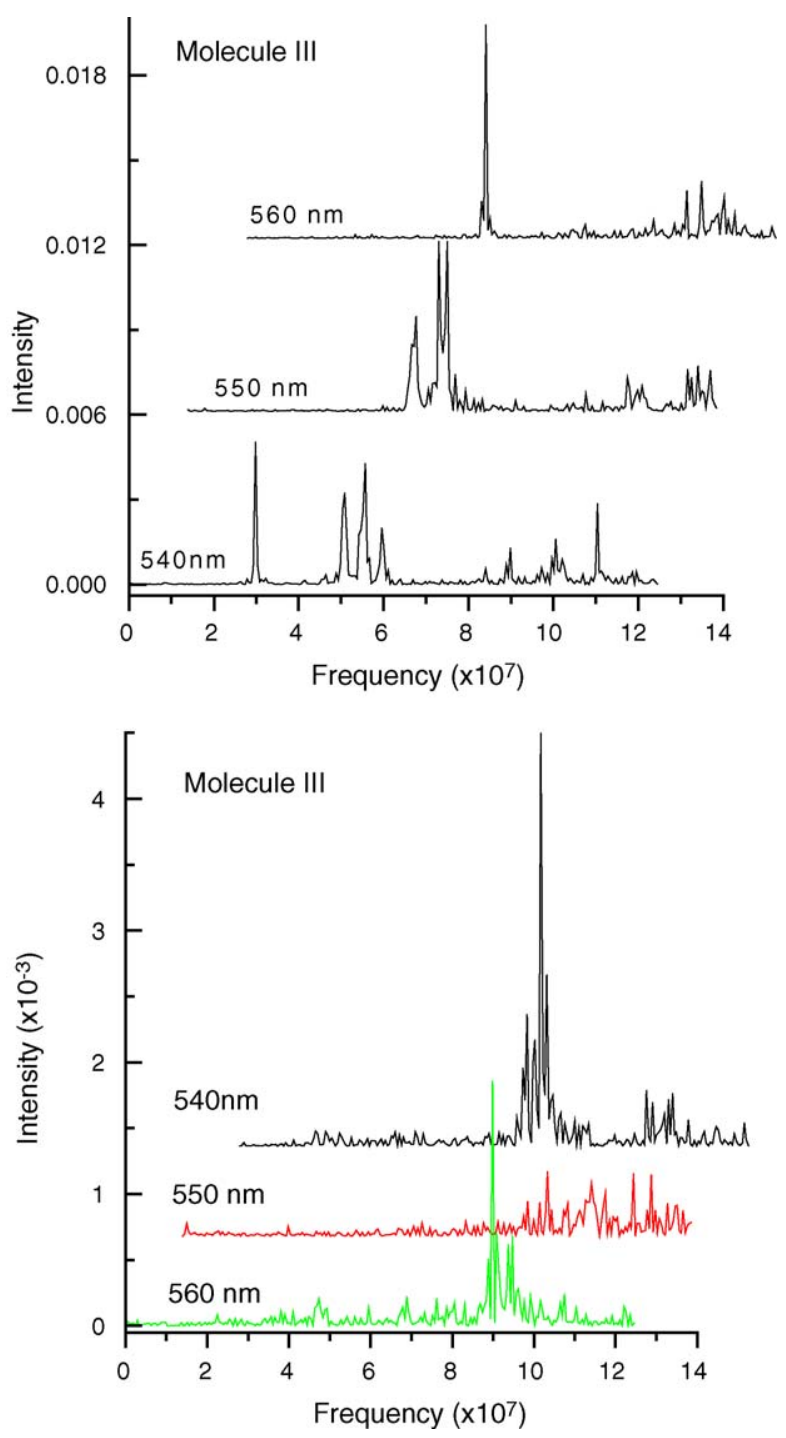

Fig. 6. The FFT results are those (above) from 0 to $2.048 \mu$ s for three wavelengths and those (below) from 34.284 to $36.332 \mu \mathrm{s}$ at $540 \mathrm{~nm}$, from 27.088 to $29.156 \mu \mathrm{s}$ at $550 \mathrm{~nm}$ and from 37.952 to $40 \mu \mathrm{s}$ at $560 \mathrm{~nm}$.

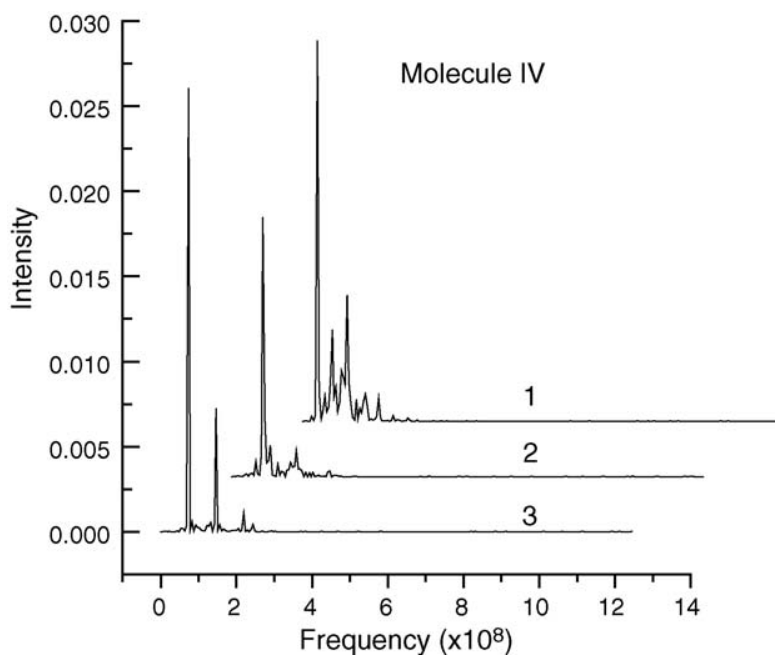

Fig. 7. The frequency domain spectra are (1) from 0 to 204.8, (2) from 3280.4 to 3484.8 and (3) from 3690.0 to $3894.4 \mathrm{~ns}$ of the time domain data at $640 \mathrm{~nm}$ for the Pt complex. 

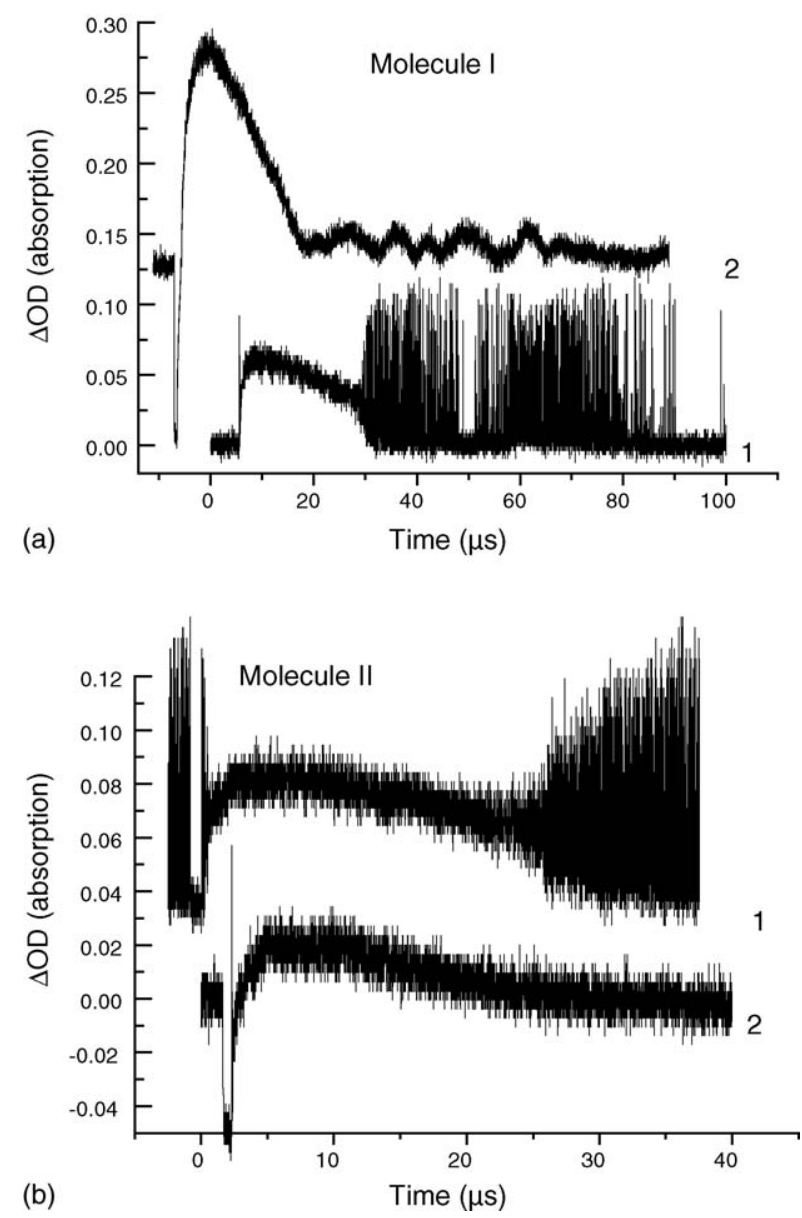

Fig. 8. The transient absorption decays of (a) adenine at $590 \mathrm{~nm}$ without (1) and with (2) static magnetic field and (b) cytosine at $590 \mathrm{~nm}$ without (1) and with (2) magnetic field.

the Pt atom valence electrons in the molecule, that is, the local electronic environment of hydrogen nuclei in the molecule has smaller change than the cases above mentioned. Hence, features of these FFT transient spectra should be nearer to the corresponding conventional ${ }^{1} \mathrm{H}$ NMR spectra, available structure-like reference such as $\mathrm{Ge}\left(\mathrm{C}_{6}-\mathrm{H}_{5}\right)_{3} \mathrm{Br}$ and $\mathrm{P}\left(p-\mathrm{FC}_{6} \mathrm{H}_{4}\right)_{3}$ [5]. Actually, all FFT spectra whose peaks lay within $2 \times 10^{8} \mathrm{~Hz}$ really supported this point during the whole time range.

The analyzed results above show that those abnormal signals are really a type of valuable spectra from samples and tentatively assigned to spin signals from excited states of molecules, such as ${ }^{1} \mathrm{H}$ NMR spectra. However, in view of the NMR principles [7], the permanent magnetic field is an essential condition. This reminds us of studying influence of the signal on an applied permanent magnetic field. In other words, if it was from magnetic spin properties of particles, there should be a significant interaction with a static magnetic field. So, a permanent static magnetic field with two magnetic bars $(1 \mathrm{~cm} \times 1 \mathrm{~cm} \times 0.5 \mathrm{~cm})$ above and below the cell was applied to same samples as shown in Fig. 2 and same LFP measurements were carried out under same other conditions. Just as our expectation, a clear effect of the abnormal signal on the permanent magnetic field could be seen as shown in Fig. 8. For adenine, abnormal phenomena disappeared completely within the measured wavelength range (200-900 nm) and the intensity of transient absorption signals increased greatly. In contrast, the lifetime decreased greatly but there seems no change for the total time of the decay. Same investigation was done for the cytosine sensitized by $\mathrm{Ru}(\mathrm{bpy}){ }_{3} \mathrm{Cl}_{2}$, and the results also show that applied permanent magnetic field could influence those transient signals but the abnormal noiselike signals still appeared in different wavelength region and also it seems to have little influence on the intensity and total time of the transient absorption decays. Of course, in both applied magnetic field experiments, the emission decays of the sensitizer $\left(\mathrm{Ru}(\mathrm{bpy})_{3} \mathrm{Cl}_{2}\right.$ ) could be detected with the transient absorption while the noise-like signals disappeared. As for the emission disappearance in the cases with noise-like, it may be neutralized results from the positive abnormal signal and negative the emission signal from the sensitizer, indicating this point in Fig. 8 (below) (1), in which the abnormal signal with a narrow blank at the earlier time stage. Same result also could be obtained from Fig. 8 (above) $620 \mathrm{~nm}$ (2) and (below) $590 \mathrm{~nm}$ (2), displaying the negative emission peaks under the absent of noise-like. But we are not clear where radio waves required occurring noise-like signals come from because there is a great energy discrepancy between the emission light and the radio wave although excited dipoles from samples may emit radio wave. Anyway, we are sure that the abnormal phenomenon results from resonance signals of nuclear or nuclear and electron with non-zero magnetic spins. For different influence of permanent magnetic field on $\mathrm{T}-\mathrm{T}$ absorption it needs further investigation in detail.

In spite of the understandable difficult in this work, we can suppose that, to some extent, some particles with magnetic spin quantum numbers also involve in the interaction between the studied sample and laser on the basis of the abnormal experimental phenomenon besides main electronic interaction. Naturally, it is most possible for these particles to be hydrogen nuclei. Under strong laser irradiation, not only a molecular electronic state can be excited but also some particle levels with magnetic nuclear spin state are split into the sublevel, which is so said Zeeman Effect, such as $+1 / 2$ and $-1 / 2$ states for hydrogen nuclear with $1 / 2$ nuclear spin quantum number. Here, due to excited states we may image that the magnetic spin particle energy level could be easier split, compared to the case in the ground state. Hence, magnetic resonance could be happened between the two levels under some suitable conditions. And strength of the signals should depend on the ratio of these magnetic spin particles to all these particles and electrons in a molecule. So, for the example molecule (IV) the proportion is relative small due to the metal atom Pt with more electrons. Therefore, its noise-like signal also is relative small. What is more, the nuclear resonance state, like the synchronized electronic excited state, also has long lifetime although the time that its level is split by laser is very short. Thus, this abnormal phenomenon perhaps is an example from optical-magnetic property, similar to the optical-electric property that we all know. However, why it appears in decay spectra with only few wavelengths should relate to some factors of the nuclear magnetic resonance absorption connecting with the decay wavelengths. Of course, these considerations about the noise-like origin are only our tentative inference. 


\section{Conclusion}

We presented some frequency spectra through analyzing the noise-like signals of four measured molecules by LFP technique. The FFT results show that molecules (I) and (IV) have respective similar spectra from the noise-like time domain signal before and after $\mathrm{T}-\mathrm{T}$ signal rising but the other two molecules containing oxygen atom have a great change between different abnormal signal time intervals, attributing to the corresponding tautomer. On the whole, no matter what one calls the abnormal signals, it at least possesses a component containing nuclear spin resonance feature. To this point, neither conventional NMR nor ESR spectra can be obtained if samples are not under a strong static magnetic field up to now. A probable origin was from hydrogen nuclear or nuclear and electron with magnetic spin quantum numbers. So, to some extent, this phenomenon indicates that protons also might play an un-negligible role in the interaction between materials and photons in some cases.

\section{References}

[1] Ian Carmichael, G.L. Hug, J. Phys. Chem. Ref. Data 15 (1986) 1.

[2] The transient absorption spectra of some Pt complex compounds were measured in author's lab for Q.Y. Cao in November 2003, who has finished his doctoral thesis Synthesis, Characterization and Photophysical Properties of Platinum(II) and Copper(I) Functional Complexes with Polypyridine Ligands at the Graduate College, The Chinese Academy of Sciences, in June 2004.

[3] Nanjing University Mathematic Group, Calculus Transform, 7th Printing, Advanced Education Press, China, 1997.

[4] G. Guelachvili (Lecture), Z.L. Zhang (Translator and Editor), Fourier Transform Spectrum, Beijing University Press, Beijing, 1990.

[5] C.J. Pouchert, The Aldrich Library of NMR Spectra, vol. 2, II ed., Aldrich Chemical Company Inc. Press, USA, 1983.

[6] E. Lippert, J.D. Macomber, Dynamics During Spectroscopic Transitions, Springer-Verlag, Berlin Heideberg, 1995, 79 (Chapter 4).

[7] Kitamaru Kamezo (Japanese writer), Q.R. Zhu (Chinese translator), Foundation and Principle of Nuclear Magnetic Resonance, Chinese Edn., University Press of Science and Technology of China, Hefei, 1991. 